\title{
Análisis de cocitación de autor en el modelo de aceptación tecnológico, 2005-2010
}

\author{
Carlos Córdoba-Cely*, Francesc Alpiste*, \\ Felipe Londoño***, Josep Monguet ${ }^{\star \star}$
}

Resumen: Este artículo explora las tendencias de investigación en el Modelo de Aceptación Tecnológico (TAM) a través del método de Análisis de Cocitación de Autor (ACA) entre los años 2005 y 2010. Por medio de la ISI Web of Knowledge (WoK) se identificaron 38 autores claves sobre los cuales se realizó un Análisis Factorial y un análisis de redes Pathfinder. El objetivo de este documento es complementar estas técnicas de visualización de conocimiento para identificar los tópicos de investigación más populares en los últimos cinco años del TAM. Los resultados muestran la existencia de dos nuevos tópicos de investigación así como la existencia de tres constructos destacados por los investigadores del TAM. Se discuten los resultados obtenidos.

Palabras claves: modelo de aceptación tecnológico (TAM), visualización de dominios de conocimiento (VKD), análisis de cocitacion de autor (ACA), redes Pathfinder (PFNET), análisis factorial.

\section{Author Co-citation Analysis of the Technology Acceptance Model, 2005-2010}

Abstract: This paper explores the research trends of the Technology Acceptance Model (TAM) using Author co-citation analysis (ACA) methods, from January 2005 to June 2010. Through the ISI Web of Knowledge (WoK) 38 key authors were selected: a Factor Analysis was performed and different techniques of information visualization, such as Multidimensional Scaling (MDS) and Pathfinder Network (PFNET), were applied. The goal of this article is to identify the most popular research topics of TAM over the five years studied. The results obtained reveal two new research themes, as well as the existence of three constructs bighlighted by researchers of TAM. The results are discussed.

Keywords: technology acceptance model (TAM), visualizing knowledge domains (VKD), author co-citation analysis (ACA), pathfinder network (PFNET), factor analysis.

* Universidad de Nariño, Colombia. Correo-e: cordobacely@udenar.edu.co.

** Universidad Politécnica de Cataluña, Barcelona. España. Correo-e: alpiste@ege.upc.edu; jm.monguet@upc.edu.

**** Universidad de Caldas, Colombia. Correo-e: felipecl@ucaldas.edu.co.

Recibido: 25-05-2011; 2. ${ }^{a}$ versión: 09-11-2011; aceptado: 21-11-2011. 


\section{Introducción}

El Modelo de aceptación tecnológico (Technology Acceptance Model, TAM) es uno de los campos de estudios más importantes en Sistemas de Información (Information System, IS) de los últimos veinte años (Bagozzi, 2007; Benbasat, 2007). Los sistemas de información se utilizan con frecuencia para referirse a la interacción entre personas, procesos algorítmicos, datos y tecnologías. Es decir, no sólo hacen referencia a las tecnologías de la información y comunicación que utiliza una organización, sino que también incluye a las personas que interactúan con estas tecnologías (Kroenke, 2009). Por esta razón, uno de los temas recurrentes de los $I S$, ha sido identificar aquellos factores que determinan el uso de un sistema entre los usuarios. Así, la aparición del Modelo de aceptación tecnológico por Fred Davis en 1985 como parte de su tesis doctoral del MIT Sloan School of Management, significó la reconfiguración de los IS hasta el punto de convertir el modelo TAM en un paradigma de clase (Bagozzi, 2007). En su propuesta, Davis (1989) parte de modelos de la psicología social como la Teoría de la Acción razonada (Fishbein y Ajzen, 1975), para proponer que la evaluación de los IS puede realizarse por medio de las características del sistema y las motivaciones de los usuarios (Chuttur, 2009). Mientras las características del sistema se explican por medio de pruebas de usabilidad (Nielsen, 1993), las motivaciones de los usuarios se explican por medio de tres factores conocidos como el núcleo del TAM: Percepción de Facilidad de Uso (PFU), Utilidad percibida (UP) e Intención de comportamiento (IC) alrededor del uso del sistema (Venkatesh y Davis, 1996). En la figura 1 se muestran las relaciones existentes entre las construcciones teóricas del modelo aquí descrito.

FIGURA 1

Modelo de aceptación tecnológico (Venkatesh y Davis, 1996)

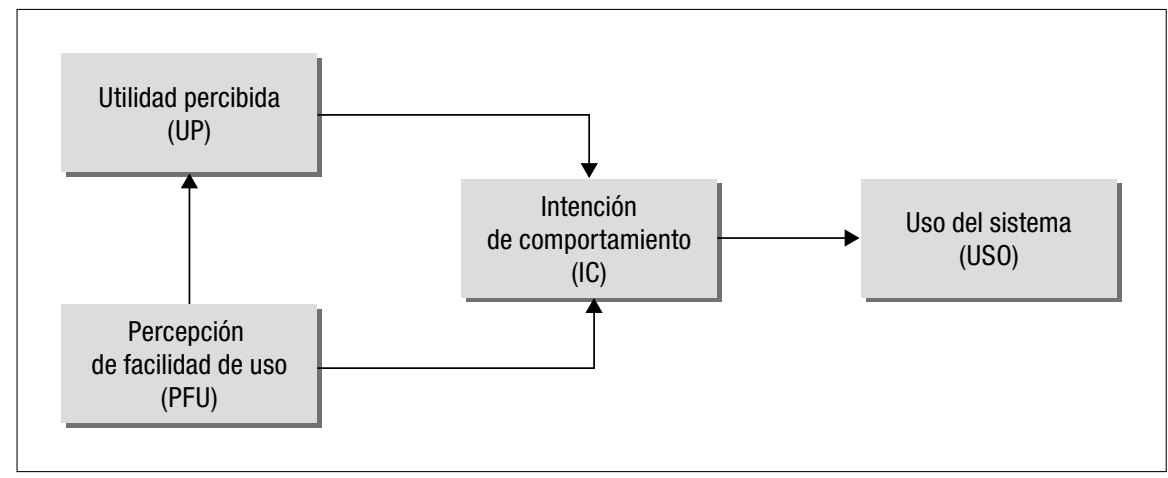

Numerosos estudios han permitido consolidar la PFU y la UP como predictores de la Intención de comportamiento del usuario (Davis y otros, 1989; Venkatesh 
y Davis, 1996; Venkatesh y otros, 2003; Chuttur, 2009), al punto de convertirse en un núcleo paradigmático sobre el cual se añaden diferentes unidades teóricas como extensiones al modelo básico (Venkatesh y Davis, 1996; King y He, 2006; Bagozzi, 2007; Benbasat, 2007). Estas adiciones al modelo básico, han perjudicado el desarrollo de una tradición de investigación y han creado caos, fragmentación y falta de integración en este campo de estudio, debido a las innumerables variaciones que sufre el modelo (Bagozzi, 2007; Benbasat, 2007). Sin embargo, al abordar el núcleo del TAM como un paradigma dominante en los $I S$, es posible identificar los temas más destacados generados por el modelo en un período de tiempo. Esta evolución cronológica, permite introducir orden en el caos y explorar el desarrollo del paradigma en términos de temáticas y autores influyentes.

Por otra parte, se entiende como paradigma a todo cuerpo de conocimiento compartido por una comunidad que da forma a una disciplina científica durante un período específico de tiempo (Kuhn, 1996). En muchas ocasiones, el conocimiento compartido del paradigma se puede representar como una red científica, una red social de coautoría o como redes de citas y cocitación de los autores (Chen, 2004). Estas redes de conocimiento compartido permiten estudiar los cambios conceptuales en el paradigma y pueden representarse por diferentes técnicas de visualización de dominios de conocimiento (Chen, 1998; Chen y Kuljis, 2003; Börner y otros, 2003). A pesar de esto, rara vez se han usado técnicas de visualización del conocimiento en el TAM (Thomson Reuters, 2009) y los investigadores han optado por otras técnicas como el meta-análisis (Legris y otros, 2003; King y He, 2006; Sun y Zhang, 2006; Schepers y Wetzels, 2007). Esto se debe a que el meta-análisis es un método de síntesis estadístico de literatura que permite resultados precisos y fiables sobre modelos teóricos (Rosenthal y DiMatteo, 2001; King y He, 2006; Schepers y Wetzels, 2007). Sin embargo, el meta-análisis depende de los criterios de inclusión/exclusión que el investigador establece al inicio de su análisis lo cual imprime una visión sesgada sobre cualquier cuerpo de conocimiento (Rosenthal y Di Matteo, 2001). Por el contrario, las técnicas de la Visualización de Dominios de Conocimiento (VKD), utilizan métodos matemáticos como el análisis de la reducción de factores y redes Pathfinder (PFNETs), las cuales permiten mostrar un enfoque de «múltiples indicadores» $y$, por tanto, mostrar una visión más amplia del paradigma estudiado (Börner y otros, 2003; McCain y otros, 2005). Así, el objetivo principal de este artículo, es encontrar los cambios más relevantes de la red de conocimiento que integra el Modelo de Aceptación Tecnológica durante los años 2005 a 2010 por medio del análisis de los siguientes indicadores: 1) autores claves del TAM; 2) nuevos temáticas de investigación sobre el TAM, y 3) construcciones teóricas más destacadas del TAM. Con el análisis de estos indicadores, se pretende obtener una visión actualizada y exhaustiva del paradigma de la aceptación tecnológica.

Así, este documento se organiza de la siguiente manera. En la sección 2, se describe la técnica bibliométrica del Análisis de Cocitación de Autor (ACA) y el procedimiento para la selección del grupo de autores clave del TAM. De igual manera, en esta sección se explica el procedimiento para identificar las construc- 
ciones teóricas más destacados del modelo. En la sección 3 se presentan los resultados del análisis factorial y las PFNETs obtenidas por medio de las matrices de cocitación. Por último, se exponen las conclusiones de los resultados obtenidos y se presentan las limitaciones de este trabajo.

\section{Análisis de cocitación de autor (ACA)}

El Análisis de Cocitación de Autor (ACA) es una técnica bibliométrica que consiste en encontrar las co-ocurrencias en la lista de referencias de artículos científicos, con el fin de caracterizar la estructura intelectual de una disciplina a partir de los autores cocitados como sustitutos de los conceptos que representan (White y Griffith, 1981; Culnan 1986; Sircar y otros, 2001; Miguel y otros, 2007). El enfoque del ACA se basa en la idea de que aquellos autores destacados con alta afinidad conceptual entre sus obras (oeuvre), tienden a ser cocitados por otros autores del mismo campo. Estos autores destacados pueden proporcionar una "visión de campo" de cualquier disciplina en un período de tiempo y determinar la tradición acumulada en un área de conocimiento (White y Griffith, 1981; Culnan 1986; McCain, 1990; Sircar y otros, 2001).

Según White y McCain (1998) el análisis de citas es el fundamento de diferentes disciplinas como la cienciometría, la bibliometría, la infometría, la cibermetría, y todas tienen un origen común en la obra pionera de Eugene Garfield (1955). Para Börner y otros (2003), la visualización de dominios de conocimiento a partir del análisis de citas, se originó con el mapeo histórico sobre la investigación en ADN (Garfield y otros, 1964) y con los trabajos de Derek Price (1961; 1965) sobre redes científicas que fueron perfeccionándose durante las décadas siguientes, mediante la visualización de "distancias" entre autores y conglomerados, al utilizar el escalamiento multidimensional y el concepto de oeuvre en sus trabajos (Small y Griffith, 1974; Griffith y otros, 1974; White, 1981; White y Griffith, 1981). Las posibilidades de ésta técnica continúan ampliándose con los trabajos de Garfield (1994), sobre mapeo longitudinal para detectar los avances de la investigación científica en un orden cronológico, y con los diferentes trabajos de Noyons sobre mapas bibliométrico para medir la similitud de publicaciones por medio palabras claves (Noyons y Van Raan, 1998; Noyons y otros, 1999; Leydesdorff y Wouters, 1999). Durante la primera década del nuevo siglo, se han dispuesto nuevas herramientas de trabajo para el ACA con la implementación de diferentes técnicas de normalización para matrices de autores y la utilización de variadas técnicas de visualización de datos como ocurre con el caso de las redes Pathfinder (Chen, 1998; White, 2003a; Chen y Kuljis, 2003; Leydesdorff, 2008; Egghe y Leydesdorff, 2009). Esta amplitud de posibilidades bibliométricas, ha permitido que el ACA pueda examinar las estructuras cognitivas de diferentes disciplinas como Ciencias de la Información (Ma y otros, 2009; Moya y otros, 2006), Gestión del Conocimiento (Chen y Lee, 2006; Ma y Yu, 2009; Pilkington y Meredith, 2009), Computación Ubicua (Lee y Chen, 2009) y diferentes temas 
médicos (Malin y Caley, 2007; Vargas-Quesada y otros, 2007; Raghupathi y Nerur, 2008), entre otros.

\subsection{Selección de autores}

Para obtener la "visión de campo" del TAM, se buscó una lista de los autores más citados con la frase Technology Acceptance Model en la ISI Web of Knowledge, y se definió el período de publicación entre enero de 2005 a junio de 2010. Este período de tiempo fue elegido para identificar la influencia de los autores noveles y sus tópicos de investigación, en un paradigma que se caracteriza por tener un gran número de variaciones del modelo original, lo cual dificulta la integración de conocimiento (Bagozzi, 2007; Benbasat, 2007). Siguiendo a Culnan (1986), se examinó la estructura intelectual global del TAM a partir de una lista con la mayor cantidad de autores sobre este tema que posteriormente se redujo a partir del número de cocitaciones, y al hecho de que todos los documentos hicieran referencia a uno de los cinco tópicos de investigación del TAM según establece King y He (2006) en su meta estudio. Así, de un total inicial de 1.275 publicaciones encontradas, se obtuvo un grupo de 81 autores seminales que fueron citados 15 veces o más durante el período de tiempo estipulado. El punto de corte de 15 citas se eligió basándose en el hecho de que la mayoría de los artículos destacados se citan dos veces al año en promedio, con una variación a positivo de este dato según la disciplina evaluada (Culnan, 1986). Para el caso

TABLA I

Autores claves del TAM

\begin{tabular}{l|l|l}
\hline Wu, J. H. (108) & Hong, S. J. (32) & Huang, H. M. (20) \\
\hline Wixon, B. H. (67) & Lai, V. S. (29) & Ngai, E. W. T (20) \\
\hline Kankanhalli, A. (54) & Brown, S. A. (26) & Sánchez-F., M. J. (18) \\
\hline Bruner, G. C. (48) & Benbasat, I. (26) & Cheng, T. C. E. (18) \\
\hline Pavlou, P. A. (47) & Sun, H. (25) & Thong, J. Y. L. (17) \\
\hline Luarn, P. (46) & Burton-Jones, A. (25) & Nelson, R. R. (17) \\
\hline Nysveen, H. (45) & Cheong, J. H. (23) & Zviran, M. (17) \\
\hline Shang, R. A. (41) & Saade, R. (22) & Ma, W. W. K. (17) \\
\hline Malhotra, N.K. (39) & Cao, M. (22) & Ong, C. S. (15) \\
\hline Kim, S. S. (33) & Lu, J. (21) & Wu, I. L. (15) \\
\hline Lee, M. K. O. (32) & Schepers, J. (21) & Ha, I. (15) \\
\hline Lin, C. S. (31) & Chiu, C. M. (20) & Turel, O. (15) \\
\hline Yu, J. (30) & Hsu, C. L. (20) & \\
\hline
\end{tabular}

Los números en paréntesis indican la frecuencia de citas de los autores durante el periodo de tiempo estipulado. 
de los $I S$, se ha utilizado con anterioridad un promedio de tres citas por año para los artículos más importantes (Sircar y otros, 2001). Del grupo de 81 autores seminales, se eliminaron de la matriz de cocitación aquellos que no mostraron correlación con ningún otro autor, así como aquellos autores que no cumplía con el punto de corte debido a que algunas de sus citas no provenían de artículos, sino de memorias de congresos o revisiones bibliográficas (White y McCain, 1998). Como se muestra en la tabla I, el grupo final de autores clave se compuso de 38 investigadores.

\subsection{Matrices de cocitación}

Sobre la lista de autores clave, se construyó una matriz de 38 × 38 para contar el número de cocitas entre cada par de autores. De acuerdo con investigaciones anteriores (Culnan 1986; White y McCain 1990; Sircar y otros, 2001; White y Griffith, 1982), el valor de la diagonal de la matriz se calculó sumando los tres datos más altos de cocitación de cada autor y dividiendo dicho resultado por dos para su análisis factorial. El proceso de normalización en el ACA ha sido largamente discutido desde que White y otros investigadores, lo incluyeron como parte de sus métodos de trabajo. Para estos autores, el coeficiente de Pearson ha representado una excelente medida de similaridad que permite identificar la cercanía de perfiles de cocitación y eliminar las diferencias entre escalas de aquellos autores que son muy citados (Griffith, 1980; White y Griffith, 1981; McCain, 1990; White y McCain, 1998; White, 2003b). A pesar de que ha sido ampliamente aceptado en el ACA como medida de similaridad, el $r$ de Pearson ha sido cuestionado como medida de distancia en la visualización de la matriz de datos debido a su sensibilidad a la inclusión de ceros en la matriz (Ahlgren y otros, 2003; Leydesdorff y Vaughan, 2006; Leydesdorff, 2008; Egghe y Leydesdorff, 2009). Para solucionar esta deficiencia, Egghe y Leydesdorff (2009) han propuesto relacionar el coeficiente de Pearson con el coseno de Salton por medio de un umbral de transformación, mientras que White (2003a), ha propuesto utilizar el algoritmo PFNET del mínimo peso entre dos puntos, por medio de la definición de los parámetros $r$ y $q$ de Chen (1998). En nuestro caso, hemos decidido: 1) utilizar el coeficiente de Pearson en el análisis factorial por su larga tradición como medida de similitud en el ACA, y 2) utilizar la matriz original con su diagonal igual a cero para la visualización de los datos por medio del algoritmo PFNET, pues su propuesta permite trabajar con la información original sin ningún tipo de normalización o umbral de transformación.

Por otra parte el ACA establece que los autores citados no son más que sustitutos de los conceptos que representan, es decir de las construcciones teóricas que conforman la oeuvre de un autor. Siguiendo este principio, es posible sustituir cada uno de los 38 autores claves por los respectivos tópicos conceptuales que ellos representan en una distribución de frecuencias, tal como se muestra en la tabla II. En psicología como en IS, cada uno de estas abstracciones teóricas 
que describe un fenómeno real no observable se denomina Constructo (Edwards y Bagozzi, 2000; Petter y otros, 2007), y su frecuencia en la tabla indica la cantidad de autores clave que han utilizado dicho argumento como parte de su propuesta teórica para explicar el TAM entre el 2005 y el 2010. Siguiendo a Edwards y Bagozzi (2000), los criterios para identificar un argumento teórico como constructo es que el término: 1) describa y asigne significado a un fenómeno de interés teórico en el TAM; 2) que el término se encuentre asociado de manera directa a un grupo de indicadores relacionados al TAM, y 3) que el término haga parte de un modelo teórico en donde se relacione con otros términos del TAM. Así, de un total de 56 Constructos válidos encontrados, se muestran los 16 más destacados teniendo como punto de corte el mismo principio utilizado por Culnan (1986) y Sircar y otros (2001) para la selección de autores seminales, es decir, el hecho de que cada entidad seleccionada aparezca por los menos tres veces en el período de tiempo estipulado.

\section{TABLA II}

Distribución de frecuencias para constructos del TAM

\begin{tabular}{|c|c|c|}
\hline Constructo (abreviatura) & Frecuencia & Frecuencia (\%) \\
\hline Percepción de utilidad (PU) & 27 & 11,15 \\
\hline Intención de comportamiento (IC) & 27 & 11,15 \\
\hline Percepción de facilidad de uso (PFU) & 26 & 10,74 \\
\hline Actitud (Att) & 16 & 6,61 \\
\hline Uso del sistema (USO) & 8 & 3,30 \\
\hline Percepción de disfrute (PD) & 8 & 2,89 \\
\hline Flujo (FL) & 7 & 2,89 \\
\hline Normas subjetivas (NS) & 7 & 2,47 \\
\hline Satisfacción (Sat) & 6 & 2,06 \\
\hline Autoeficacia (AE) & 5 & 2,06 \\
\hline Intención continuada de uso (ICU) & 5 & 1,65 \\
\hline Género/Edad (G/E) & 4 & 1,65 \\
\hline Calidad del sistema (CS) & 4 & 1,65 \\
\hline Información del sistema (IS) & 4 & 1,65 \\
\hline Confirmación (Con) & 4 & 1,65 \\
\hline Confianza (Conz) & 3 & 1,23 \\
\hline
\end{tabular}

Estas construcciones teóricas destacadas pueden organizarse en una matriz de $16 \times 16$ para encontrar las respectivas cocitaciones entre ellas. Para ello, se agrupa a los autores claves de cada constructo y se cruzan con los respectivos autores de los restantes constructos de la matriz. Por ejemplo, tal como se muestra 
en la tabla III, para obtener las cocitaciones entre los constructos de confirmación y confianza, se cruzaron los cuatro autores que han utilizado el término confirmación (Chiu, Hong, Lin, Thong) con los tres autores que han utilizado el término confianza ( $\mathrm{Yu}$, Wu I. L., Wu, J. H.), dando como resultado 18 cocitaciones totales. Para determinar si esta matriz es válida como escala de medida para construir redes, es necesario aplicar un coeficiente de psicometría como el Alfa de Cronbach que permita cuantificar la consistencia interna para cada uno de los 16 constructos a partir de las varianzas obtenidas de las cocitaciones totales de cada ítem. Dentro de la categoría de coeficientes psicométricos, el Alfa de Cronbach es el más utilizado por los investigadores, pues con la administración de una única prueba es posible estimar el límite inferior del coeficiente de fiabilidad de un grupo de constructos (Ledezma y otros, 2002). Al introducir los datos de los 16 constructos al software SPSS, se obtuvo un Alfa de Cronbach de 0.893, lo cual indica una alta confiabilidad para utilizar estos términos teóricos como escala de medida para construir redes.

TABLA III

Matriz parcial de cocitación de constructos por medio de autores. Ejemplos de cocitación entre constructos de Confirmación $\times$ Confianza en el TAM

\begin{tabular}{c|l|c|c|c|c}
\hline \multicolumn{2}{c}{} & \multicolumn{5}{c}{ Confirmación } \\
\cline { 3 - 6 } \multicolumn{2}{c}{} & Chiu & Hong & Lin & Thong \\
\hline \multirow{3}{*}{ Confianza } & 0 & 1 & 4 & 2 \\
\cline { 2 - 6 } & Yu & 0 & 1 & 2 & 0 \\
\cline { 2 - 6 } & Wu, I. L. J. H. & 1 & 2 & 4 & 1 \\
\hline
\end{tabular}

\section{Análisis de datos}

El Análisis factorial se obtuvo con la correlación de Pearson de los autores utilizando el programa SPSS versión 17.0. Las PFNETs se derivaron de los datos originales obtenidos en las cocitaciones por medio del programa PCKnot. El programa UCINET versión 6.0, se utilizó para medir la intermediación de flujo en las redes, y el programa Pajek versión 1.28 fue utilizado para la visualización gráfica. Por último, las redes se graficaron mediante CS5 Illustrator para agrupar los datos y mejorar los gráficos presentados en este documento. De igual manera, para interpretar adecuadamente los resultados, se modificaron los tamaños de los nodos y los espesores de las líneas en todas las imágenes.

\subsection{Análisis factorial}

La técnica de Análisis factorial se utiliza para reducir las variables originales de un problema en un número menor de dimensiones o factores, en donde cada 
factor se explica por aquellas variables que cargan sobre él (McCain, 1990; Sircar y otros, 2001). Esta reducción dimensional ha permitido que el Análisis Factorial sea una técnica ampliamente utilizada en la metodología tradicional del ACA junto con el Análisis de Conglomerados y el Escalamiento Multidimensional. Usando una rotación Varimax y una normalización Kaiser sobre los 38 autores claves, se presentan en la tabla IV los principales componentes obtenidos con este procedimiento. Siguiendo a McCain (1990) se muestran los autores con una carga superior a $\pm 0,4$, aunque para la interpretación de cada factor sólo se utilizan las cargas superiores a $\pm 0,7$. Debido a que el Análisis Factorial permite extraer tantos factores como variables pueda tener un problema, McCain (1990) sugiere que para el ACA dicha extracción se detenga cuando el eigenvalue sea menor a uno $(<1)$. Así, los primeros 8 factores extraídos haciendo el eigenvalue $<1$ dan como resultado una varianza acumulada de $77,6 \%$ similar a los resultados obtenidos en otros estudios en IS (Culnan, 1986; Sircar y otros 2001; White, 2003a). El factor 9, con 3,4\% de la varianza, se abandonó debido a la dificultad de conciliar los tema de investigación entre los dos autores que cargaban sobre este tema. Estos autores (Kankanhalli y Zviran) encuentran su conglomerado correspondiente en el análisis de PFNETs.

Los autores que cargan sobre el factor 1 basan su investigación en Tecnologías móviles. Este factor incluye tópicos relacionados con negocios móviles (Luarn y Lin, 2005), evaluación de Sistemas de información aplicados a tecnologías móviles (Wu y Wang, 2005), m-commerce (Bruner y Kumar, 2005; Cheong y Park, 2005), y comportamiento e influencia de los servicios móviles (Nysveen y otros, 2005). El factor 2 explica las diferentes características de la post-adopción tecnológica por medio de la adaptación del modelo de Expectación-Confirmación como ocurre con Lin y otros (2005), y Hong y otros (2006), o ya sea por medio de nuevos modelos como sucede con Kim y Malhotra (2005) con su modelo longitudinal continuado. El factor 3 aparece definido como Motivaciones del usuario y el factor 4 como Validación de la calidad. Así, los autores reunidos en el factor 3 trabajan con tópicos basados en los Modelos motivacionales (Shang y otros, 2005), la Absorción cognitiva (Saadé y Bahli 2005) y el Flujo (Sanchez-Franco y Roldán, 2005) como variables de interpretación, mientras que los autores agrupados en el factor 4 centran su trabajo en la validación de la calidad de los sistemas y la calidad de la información por medio de las variables de percepción del comportamiento de Control (Wixon y Todd, 2005; Cao y otros, 2005). Por otra parte, mientras el factor 5 incluye autores como Brown y otros (2006) y Ha y otros (2007), los cuales estudian la adopción de las Tecnologías de la Información en el Hogar y las Aplicaciones Multipropósito como ocurre con Turel y otros (2007), el factor 6 incluye aquellos autores que realizan Contribuciones Teóricas al modelo general del TAM como sucede con Burton-Jones y Straub (2006). Por último, los autores del factor 7 se concentran en los estudios de E-learning (Ngai y otros, 2007), y los autores del factor 8 lo hacen sobre Banca en Internet (Lai y Li, 2005). 


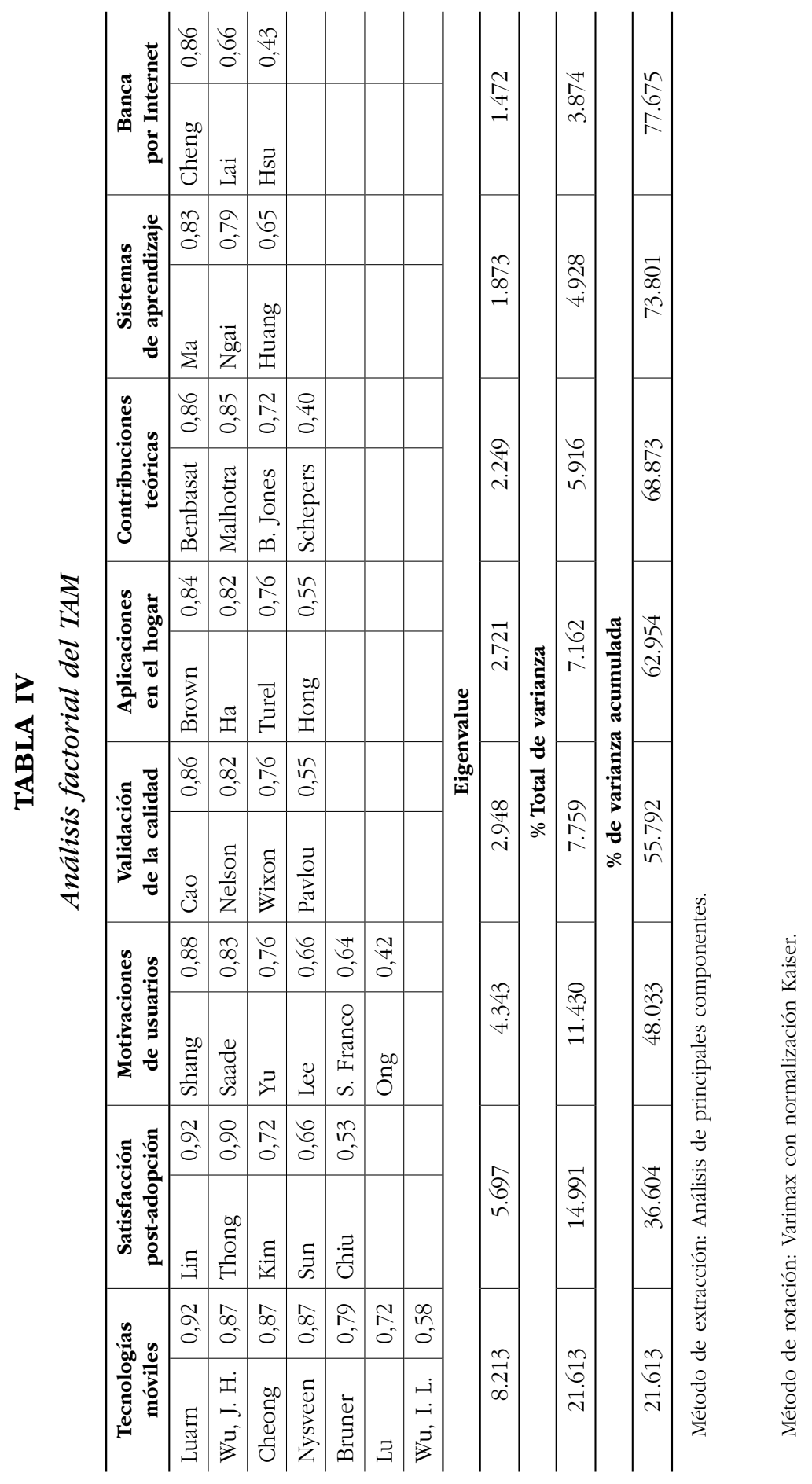




\subsection{Redes Pathfinder (PFNET)}

El algoritmo de escalamiento de redes Pathfinder (PFNET), es una técnica que extrae los patrones subyacentes en datos próximos y los representa gráficamente por medio de una red interconectada. Como ya se ha mencionado, el algoritmo PFNET tiene varias ventajas al momento de visualizar datos, pues trabaja con la información original sin ningún tipo de normalización o umbral de transformación, y permite "podar» una red compleja de múltiples vínculos usando el mínimo peso de un camino entre nodos (White, 2003a). Debido a que el peso de un camino puede ser determinado por los datos originales de la matriz de cocitación o por la matriz de correlación de Pearson (Chen y Lee 2006; Nooy y otros, 2005; White 2003b), en este estudio, se ha hecho el camino igual al número de cocitaciones originales entre autores o constructos, y los nodos iguales a los mismos autores o constructos. Por otra parte, se debe tener en cuenta que este procedimiento de poda permite destacar los caminos más importantes entre nodos y reducir los vínculos cruzados para mejorar la claridad de la visualización de la red resultante al variar la topología de los parámetros $r$ y $q$ del algoritmo PFNET (Chen, 1998; 2004). Por lo tanto, para podar las redes del TAM se procedió a utilizar el software PCKnot versión 6.3 haciendo $q=n-1$ y $r=\infty$, de la que resultaron una serie de redes en la que todos los autores y constructos claves se encuentran conectados. El parámetro $q$ limita el coste mínimo de un camino dentro del algoritmo, y el parámetro $r$ define la distancia de un camino a través de la métrica de Minkowski. De un total de 775 vínculos iniciales en la PFNET de autores, se obtuvo una red final de 36 caminos que fue graficada utilizando el algoritmo Kamada-Kawai embebido en el software Pajek versión 1.28. Para el caso de la PFNET sobre constructos, se obtuvieron 34 caminos finales de un total inicial de 258 vínculos entre nodos.

Para interpretar las PFNETs, se reponderó el espesor de las líneas, es decir los vínculos entre nodos, de acuerdo al número de cocitaciones. Adicionalmente, se varió las formas de las líneas y el tamaño de los nodos por medio de la distinción entre vínculos primarios y secundarios, y la utilización del índice de Intermediación de Flujo. De acuerdo con Bruer (2010), el algoritmo PFNET funciona adicionando vínculos a la red a medida que decrece el número de cocitaciones en el conglomerado. Esta propiedad, permite distinguir los enlaces primarios de la red y mejorar la interpretación de las relaciones entre actores y sus enlaces secundarios. Por otra parte, el análisis de un conglomerado se fundamenta en el poder social de sus actores y en las relaciones que se establecen en dicho grupo (Hanneman y Riddle, 2005). De la variada cantidad de medidas para determinar la importancia de estas relaciones, en este estudio se trabajará con dos índices específicos: la intermediación de flujo y el grado de centralidad. La intermediación de flujo mide la capacidad en que un nodo contribuye a la suma del flujo máximo de información entre todos los pares de nodos de la red. Tal como lo concibe Freeman y otros (1991) la intermediación de flujo se define por el supuesto de que cada enlace entre nodos es como un tubo que puede 
transportar una cantidad unitaria de flujo. Así, si un nodo genérico $j$ es la fuente de flujo y un nodo genérico $k$ es el objetivo de recepción, es posible calcular el caudal máximo entre ellos de la siguiente manera:

$$
C_{F i}=\frac{\sum_{j<k}^{n} m_{j k}(i)}{\sum_{j<k}^{n} m_{j k}}
$$

donde $C_{F i}$ es la intermediación de flujo en el nodo $i, \mathrm{y} m_{j k}(i)$ es la cantidad de flujo que pasa a través de $i$ cuando el máximo flujo de $m_{j k}$ se mueve de $j$ a $k$. Este índice es utilizado para intenta superar las restricciones que ofrece el grado de intermediación, el cual limita el flujo de la comunicación entre los actores de una red al camino más corto entre dos nodos (geodésica). Como explica Hanneman y Riddle (2005), esta diferencia, permite que el grado de intermediación identifique los autores con mayor poder en la red mientras que la intermediación de flujo destaque los enlaces más importante que unen diferentes nodos. Así, mientras la intermediación de flujo se calcula a partir de todos los caminos independientes entre dos puntos de la red (Nooy y otros, 2005), el grado de Intermediación se centra exclusivamente en la geodésica que incluye cada vértice en un proceso de selección binario que ha sido criticado por muchos (Freeman y otros, 1991; Peay, 1976; Yan, 1988). Aunque es evidente que los actores con mayor poder posiblemente tendrán mayor peso en sus enlaces, lo cierto es que la interpretación de una red será diferente si se hace por medio del poder del nodo o el peso del enlace. Para el caso de este estudio, se ha decidido trabajar con el peso del enlace, por medio de la intermediación de flujo, y dejar la evaluación del poder del nodo al índice de grado de centralidad.

El grado de centralidad de un vértice, es la primera de las tres medida más importantes introducidas por Linton Freeman para medir la centralidad de los actores en una red, y se define como el número de vínculos que inciden sobre un nodo específico (Freeman, 1979; Nooy y otros, 2005). Se ha determinado trabajar con esta medida porque por medio de ella es posible encontrar aquellos actores más importantes para la red según su posición de privilegio con respecto a los demás integrantes del conglomerado (Hanneman y Riddle, 2005), y complementar sus resultados con la información obtenida del índice de intermediación de flujo.

\section{PFNET de autores}

La figura 2 muestra la PFNET de los autores claves del TAM entre los años 2005 y 2010. Los vínculos primarios son líneas continuas y los vínculos secundarios son líneas discontinuas. Los autores se representan como puntos rellenos cuya importancia se expresa por el diámetro de los círculos adscritos a cada vértice según el valor de la intermediación de flujo para cada autor. La figura 2 
también muestra la división interna de la PFNET. Los conglomerados centrales de la red, aparecen separados de los conglomerados periféricos por líneas secundarias discontinuas. El conglomerado más central es el de Tecnologías móviles, y lo acompañan en importancia los conglomerados de Motivaciones de los usuarios, Aplicaciones en el hogar y Banca en Internet.

FIGURA 2

PFNET del TAM entre los años 2005 a 2010

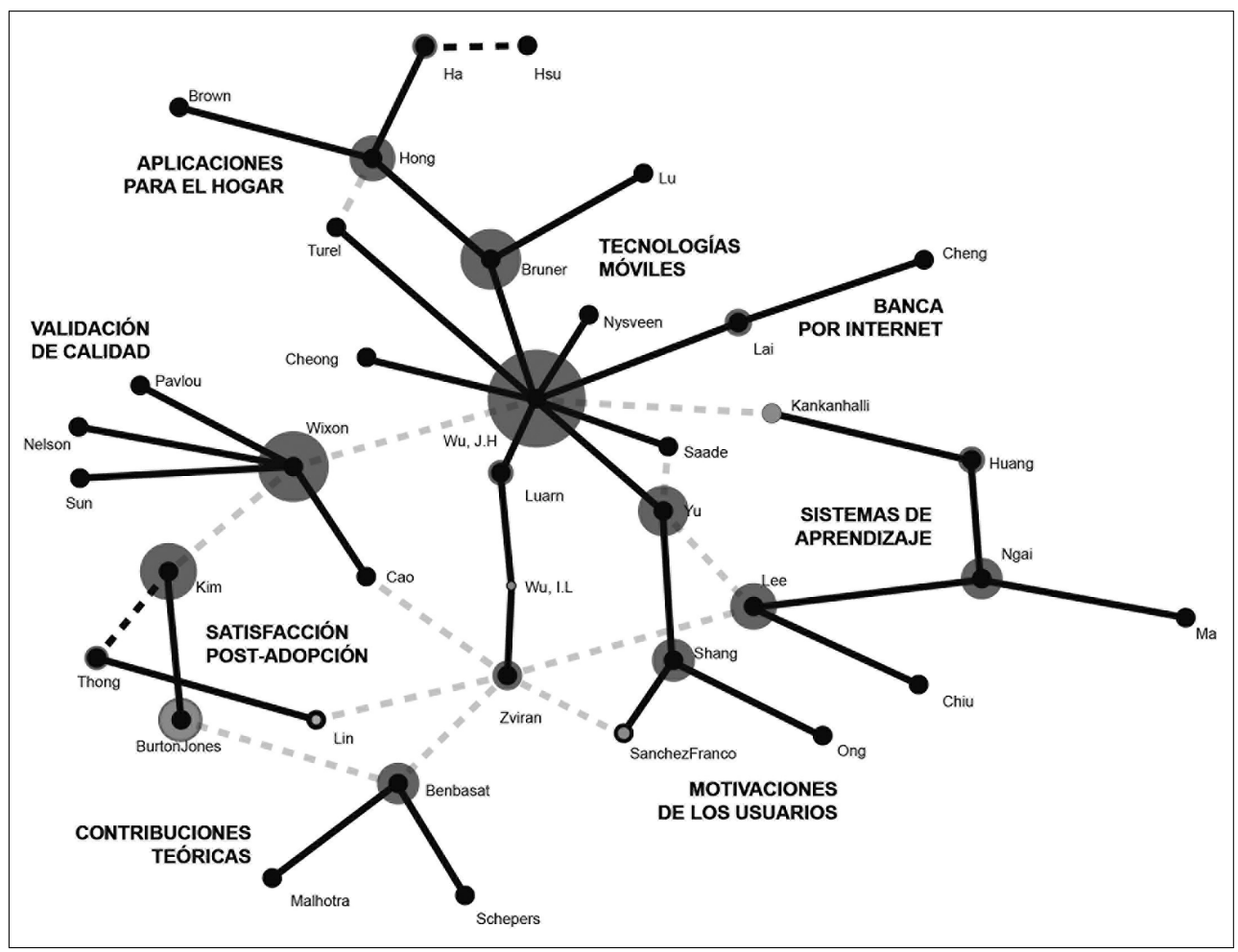

La tabla $\mathrm{V}$ muestra las medidas de grado de centralidad y de intermediación de flujo para los autores más destacados. Sobresale Wu, J. H., como el autor más importante de los últimos cinco años. Se puede observar que todos los conglomerados cuentan con al menos un autor con una alta intermediación de flujo. Así, los actores con mayor intermediación de flujo en la PFNET se pueden considerar como los autores fundacionales de cada uno de los conglomerados que originan, mientras que los autores con mayor carga factorial se pueden considerar como los más destacados en cada factor. Ya que el TAM se analiza bajo el enfoque de paradigma, es interesante notar que en ninguno de los conglomerados de la red aparecen los autores fundacionales del TAM, es decir, Davis, 
Bagozzi y Venkatesh, lo cual supone una renovación teórica sobre los fundamentos del modelo. Un ejemplo claro de este cambio, lo encara el conglomerado de Contribuciones teóricas, donde aparece Burton-Jones y Straub (2006) y Benbasat (2007), los cuales centran sus posiciones teóricas sobre el replanteamiento del modelo a partir del Uso del sistema y las Motivaciones intrínsecas del usuario.

TABLA V

Medidas de centralidad en autores claves del TAM

\begin{tabular}{l|c|l|c}
\hline \multicolumn{1}{c|}{ Autor } & $\begin{array}{c}\text { Grado } \\
\text { de centralidad }\end{array}$ & \multicolumn{1}{c|}{ Autor } & $\begin{array}{c}\text { Intermediación } \\
\text { de flujo }\end{array}$ \\
\hline Wu, J. H. & 27,02 & Wu, J. H. & 67,79 \\
\hline Zviran & 18,91 & Wixon & 37,21 \\
\hline Wixon & 16,21 & Bruner & 24,62 \\
\hline Shang & 10,81 & Kim & 24,10 \\
\hline Lee & 10,81 & Yu & 18,44 \\
\hline Bruner & 10,81 & Lee & 16,15 \\
\hline Hong & 10,81 & Hong & 15,61 \\
\hline Yu & 10,81 & Burton-Jones & 12,48 \\
\hline Wu, J. H. & 10,81 & Ngai & 11,89 \\
\hline Zviran & 10,81 & Benbasat & 11,64 \\
\hline
\end{tabular}

Por otra parte, cuando estos conglomerados son visualizados en el cambio del tiempo, es posible hablar de Continentes Intelectuales, ya que permiten mostrar los movimientos que envuelven el paradigma en un período de tiempo donde se contrastan sus cambio (Chen y Kuljis, 2003; Chen, 2004). Siguiendo esta metáfora visual, en la figura 3 se puede observar los movimientos intelectuales ocurridos entre dos períodos: los años 2005-2007 aparecen con sus líneas en color negro, y los años 2007-2010 aparecen con sus líneas en color gris. El espesor de las líneas viene dado por Pajek y está determinado por el número de cocitaciones entre autores.

La figura muestra la aparición de dos nuevos conglomerados entre los años 2007 y 2010: Aplicaciones en el hogar y Banca en Internet. El vínculo de mayor crecimiento es el que se establece entre Wu, J. H. y Luarn. De hecho, todos los vínculos alrededor de Wu, J. H., crecen entre el 2005 y el 2010. Esto convierte al conglomerado en Tecnologías móviles como el de mayor crecimiento de los últimos cinco años. Por otra parte, los conglomerados con variaciones internas de sus vínculos más sobresalientes son el de Sistemas de aprendizaje y el de Motivaciones del usuario, al adicionar los autores Lee y Shang a partir del año 2007. Ya que Lee y otros (2005) trabajan sobre el Modelo motivacional en el 


\section{FIGURA 3}

PFNET del TAM en dos períodos de tiempo. En negro vínculos entre 2005-2007 y en gris vínculos del 2007-2010

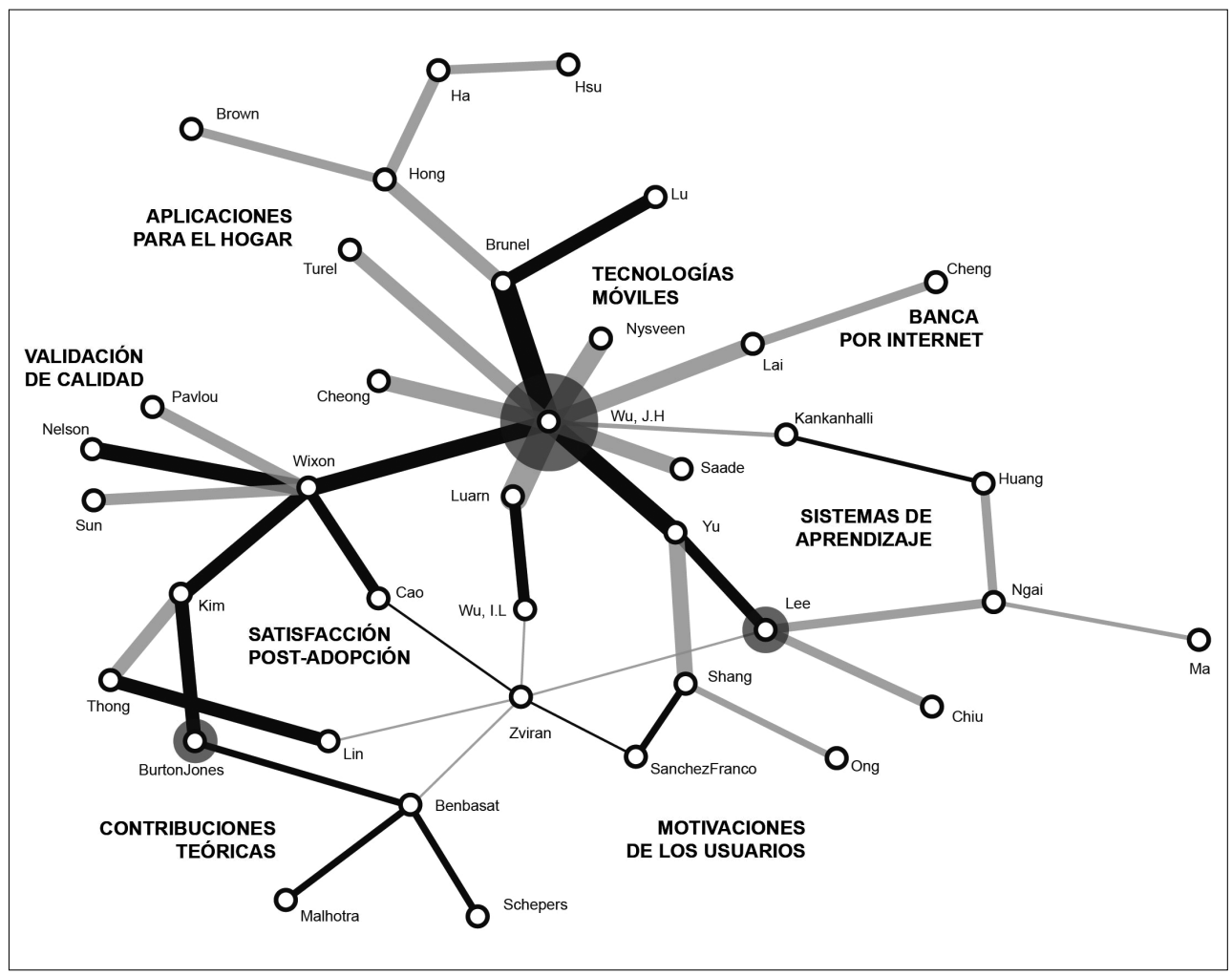

aprendizaje medio basado en internet, y Shang y otros (2005) centran su trabajo sobre las motivaciones extrínsecas e intrínsecas en compras online, se puede concluir que en la actualidad las investigaciones sobre Sistemas de Aprendizaje y Motivaciones de los Usuarios tienden a valorar las características holísticas del sistema. Es importante destacar que las anteriores PFNETs presentan bucles alrededor del autor Zviran a pesar de haber hecho $q=n-1$ y $r=\infty$, en el software PCKnot. Esto se debe al hecho de que todos los enlaces alrededor de este autor tienen el mismo número de cocitaciones (1 cocitación por cada enlace a otro autor), razón por la cual se muestra este efecto en las redes aquí expuestas. Es posible que por este motivo sea difícil ubicar a este autor en el análisis factorial, al igual que ocurre con Kankanhalli. En estos dos casos, la distinción entre enlaces primarios y secundarios marca la posición del autor en su respectivo conglomerado. 


\section{PFNET de constructos}

Para graficar las relaciones entre las construcciones teóricas del TAM, es necesario trabajar a partir de la matriz de $16 \times 16$ sobre constructos que se desarrolló con anterioridad. En la figura 4 se puede observar la red podada después de hacer $q=n-1$ y $r=\infty$, a esta matriz. Con el resultado obtenido, se observa que la relación más citada y estable de todo el TAM es la dupla Utilidad-Intención de Comportamiento con 1.295 cocitaciones, lo que convierte a este vínculo en el verdadero núcleo paradigmático del modelo. A su vez, el constructo con mayor influencia en todo el modelo es la Intención de Uso, tanto en su grado de centralidad como en su intermediación de flujo. Entre paréntesis se muestra la intermediación de flujo obtenida para cada constructo antes de realizar la poda de la red.

FIGURA 4

PFNET de constructos del TAM

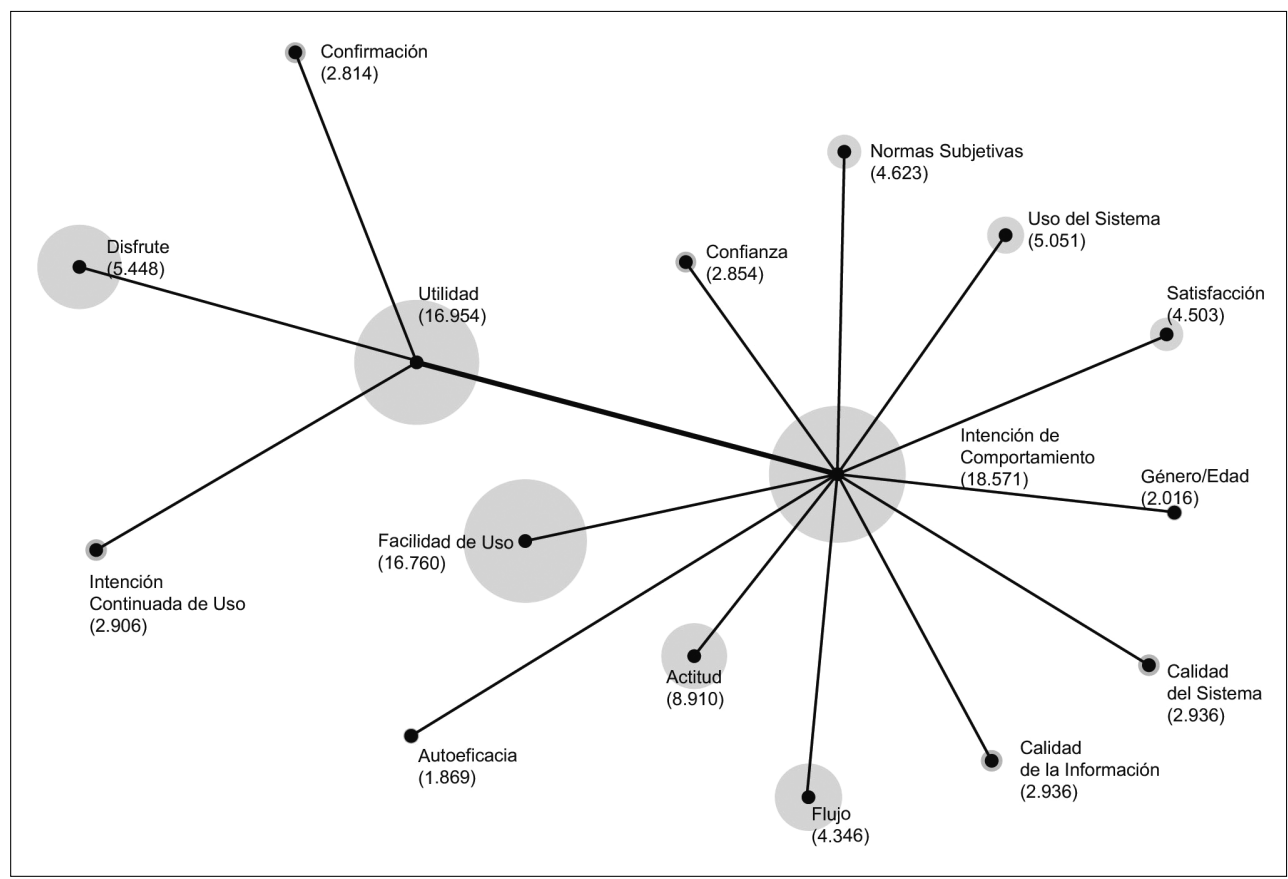

A pesar de que diferentes constructos como Confianza, Normas Subjetivas, Confirmación e Intención de Uso continuo aparecen vinculados primero a la Utilidad del sistema entre los años 2005 a 2007 y después a la Intención de Comportamiento del usuario entre los años 2007 a 2010, esta PFNET podada no permite visualizar adecuadamente estas variaciones entre los enlaces de los cons- 
tructos. Por tal razón, se presenta la figura 5, donde se aprecian las variaciones de la intermediación de flujo de los constructos es dos períodos de tiempo: En color negro se muestran los nodos del período 2005-2007, y en color gris se muestran los nodos del período 2007-2010. El constructo con mayor Intermediación de flujo de los dos períodos, es la Percepción del disfrute. Los segundos constructos con mayor Intermediación de flujo son la Norma subjetiva para el período 2005-2007, y el Uso del sistema para el período 2007-2010. Son estos tres constructos los más importantes utilizados por la comunidad del TAM en los últimos cinco años, y sobre los cuales se centra la investigación de este paradigma en la actualidad. Es claro que los modelos del TAM han evolucionado del análisis de las funciones utilitarias del sistema, como la Facilidad de Uso, la Utilidad, y la Intención de Comportamiento, a centrarse en el análisis de la experiencia del usuario como la Percepción del disfrute, y las Normas subjetivas. Para el caso del Uso del Sistema, se presenta una evolución del constructo hacia indicadores holísticos que incluyen evaluaciones más amplias que el simple desempeño de la tarea, como ocurre con la propuesta de Burton-Jones \& Straub (2006).

\section{FIGURA 5}

Intermediación de Flujo de constructos del TAM. En negro Intermediación de flujo entre los años 2005-2007, y en gris Intermediación de flujo entre los años 2007-2010]

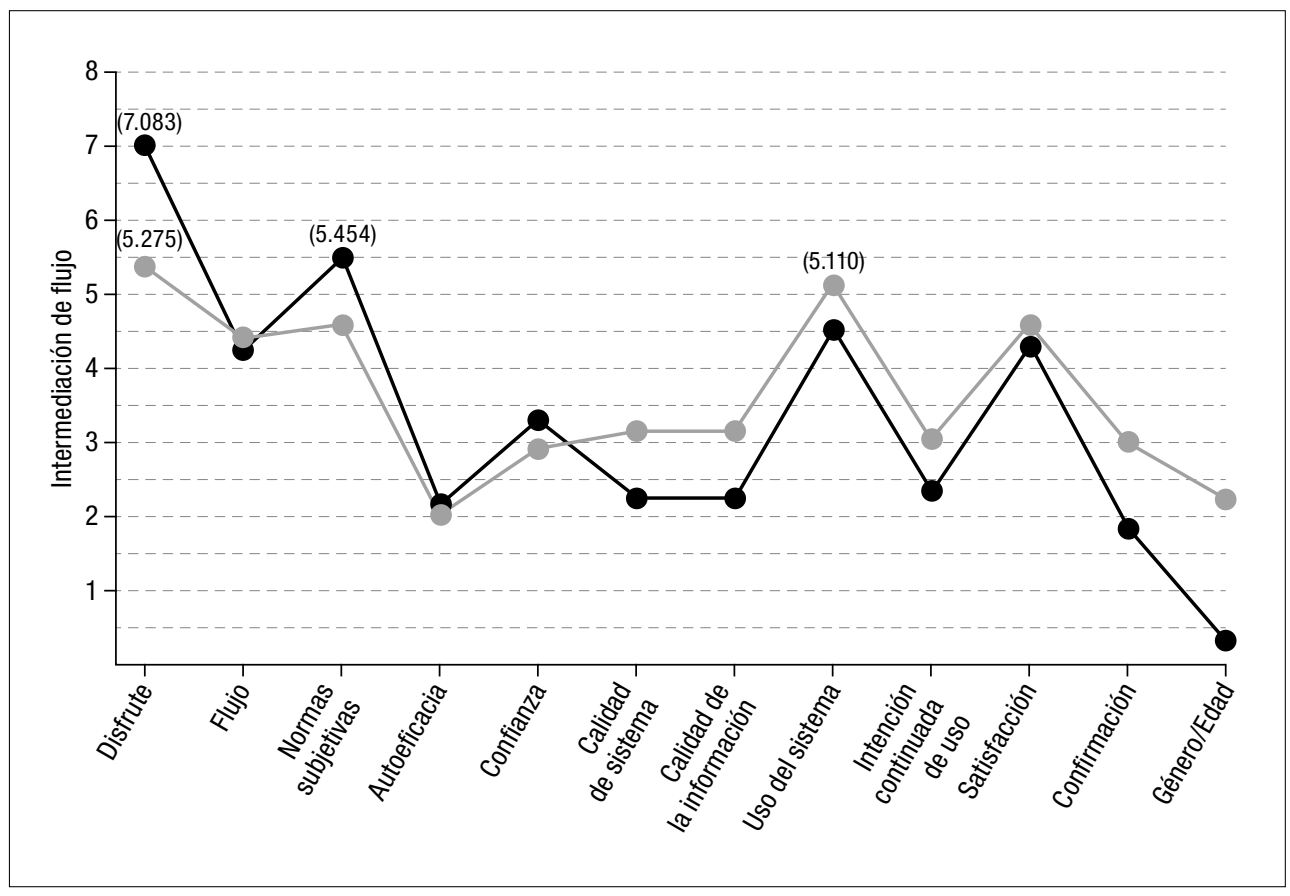




\section{Conclusiones}

Siguiendo el principio de Henderson y Clark (1990), es posible decir que la evolución del paradigma del TAM se fundamenta sobre un enfoque de Innovación Arquitectónica, es decir sobre el cambio continuo de las relaciones entre los diferentes constructos que constituyen el modelo. Esta hipótesis epistemológica puede comprobarse empíricamente por medio de las relaciones entre autores, conglomerados y constructos de los últimos cinco años, como se muestra en este estudio. Podemos decir entonces, que la innovación arquitectónica en el TAM ha pasado de una investigación centrada en objetivos utilitarios (Facilidad de Uso, Utilidad, Intención de Comportamiento, Uso del Sistema) a una investigación de tipo holística, orientada en la experiencia de una actividad (Disfrute, Actitud, Uso del Sistema), y su relación con los objetivos utilitarios del sistema. Destacan en este aspecto, los trabajos fundacionales de Wu y Wang (2005), Shang y otros (2005), Hong y otros (2006), y Lai y Li (2005). Además, se puede afirmar que la percepción de Disfrute, las Normas Subjetivas y el Uso del Sistema son los constructos más utilizados por los investigadores para predecir la aceptación tecnológica. La búsqueda de construcciones teóricas que perfilen mejor una explicación holística del modelo, ha hecho que el núcleo del TAM se complemente por medio de la Percepción de Disfrute y el Uso del Sistema, como lo demuestran los trabajos de Sanchez-Franco y Roldán (2005) y Burton-Jones y Straub (2006).

Por otra parte, los datos obtenidos en este estudio, muestran la existencia de un grupo de autores bastante heterogéneo alrededor del TAM. La razón de esta variedad se debe en gran parte a la parsimonia del modelo (Bagozzi, 2007), es decir, a la facilidad de predicción de tiene un modelo a partir de unas pocas variables, como ocurre con el núcleo del TAM y sus tres constructos bases. Esta fortaleza, ha convertido el modelo de aceptación tecnológico en una herramienta versátil para los $I S$, que ha permitido su adaptación a nuevos tópicos como Tecnologías Móviles o Banca en Internet, sin dejar de lado la predicción de sistemas tradicionales como los presentes en el Aprendizaje. Este hecho también puede explicar la escasa colaboración que existe entre autores claves (sólo se han encontrado tres relaciones: Naresh Malhotra y Sung Kim; See-Joon Hong y James Thong; Susan Brown y Viswanath Venkatesh), si se parte del principio que cada investigador busca adaptar el TAM para predecir un sistema particular con una necesidad y una población de estudio específica. Sin embargo, este tipo de conclusiones sólo se pueden asumir llevando a cabo un estudio cuyo objetivo fundamental sea este tipo de relaciones.

\section{Limitaciones}

A pesar de que el ACA es una técnica bibliométrica ampliamente utilizada, es importante mencionar algunas de sus limitaciones para complementar las conclusiones de este estudio. Posiblemente la primera limitación sea el hecho de que 
sólo se utilizan los primeros autores para la configuración de la matriz de cocitación, lo cual elimina de tajo la información sobre la colaboración entre investigadores y la configuración interna y jerárquica de equipos de trabajo. Esta deficiencia se ha visto compensada por la utilización de métodos de recopilación de datos más complejos como el Complete Author Pair (Su y otros, 2009), que a pesar de todo no resuelven el problema fundamental sobre la inclusión de todos los autores en la matriz de cocitación. Otra de las limitaciones más discutidas, se encuentra en la desambiguación de autores con idénticos nombres, ya sea dada por semejanza ortográfica o existencia de homónimos. A pesar de existir múltiples técnicas que en la actualidad solventan parcialmente esta limitación (para mayor información sobre este tema remitirse a: Kang y otros, 2009), lo cierto es que la discusión sobre este tema todavía sigue siendo «un problema sin resolver» (Tang y Walsh, 2010), que para el caso de este estudio se asumió mediante un procedimiento de desambiguación manual entre los autores claves.

Por otra parte, hay que señalar el debate relacionado con la utilización del coeficiente de Pearson en el Análisis Factorial. Debido a que el $r$ de Pearson es utilizado para distribuciones paramétricas, su utilización en la cocitación de autores ha sido criticada debido a la sensibilidad de este coeficiente a las distribuciones no-paramétricas (para mayor información sobre este tema remitirse a: Ahlgren y otros, 2003; Leydesdorff y Vaughan, 2006). Sin embargo, existe la convicción por parte de otros investigadores de que ésta técnica paramétrica proporciona distribuciones lo suficientemente robustas para no necesitar ningún tipo de transformación logarítmica, tal como se ha hecho en este estudio (para mayor información sobre este tema remitirse a: White, 2003b; Bensman, 2004). Adicionalmente es importante señalar que a pesar de que la varianza obtenida en este estudio (77\% para 8 factores) pueda parecer bastante limitada, Howard White (2003) establece que un valor entre 0,70 y 0,90 indica que «una multitud de obras de autores pueden agruparse en un pequeño número de categorías». Esto indica un posible rango de validación para la varianza acumulada teniendo en cuenta el eigenvalue $<1$.

Para finalizar, cabe destacar que el método utilizado aquí para obtener la cocitación de constructos, no se encuentra estandarizado. Se podría pensar que la cocitación de documento habría sido un mejor procedimiento de análisis para los constructos, pero entonces hubiera sido necesario realizar una PFNET para cada una de las 16 palabras claves encontradas, obteniendo de esta manera una visión parcializada de las relaciones particulares del TAM, y haciendo imposible conseguir medidas comunes de intermediacion de flujo para todos los constructos. Sin embargo, el principio fundamental de la cocitación es que los autores son sustitutos de los conceptos que representan. Por lo tanto, creemos que las características aditivas y particulares del TAM permiten la identificación de patrones para ciertas construcciones teóricas como los que aquí se presentan. Inferimos que esta creencia es posible sobre el resultado del Alfa de Cronbach (0.893) que confirma la fiabilidad de los constructos conseguidos como escala de medida para construir redes a partir de las cocitaciones originales de los autores. 


\section{Bibliografía}

Ahlgren, P.; Jarneving, B.; Rousseau, R. (2003). Requirements for a cocitation similarity measure with special reference to Pearson's correlation coefficient. Journal of the American Society for Information Science and Technology, 54, 550-560.

Bagozzi, R. (2007). The legacy of the Technology Acceptance Model and a Proposal for a Paradigm Shift. Journal of the Association for Information System, 8 (4), 244-254.

Benbasat, I. (2007). Quo Vadis, TAM? Journal of the Association for Information System, 8 (4), 211-218.

Bensman, S. (2004). Pearson's $r$ and author co-citation analysis: A commentary on the controversy. Journal of the American Society for Information Science and Technology, 55 (10), 935-936.

Brown, S. A.; Venkatesh, V.; Bala, H. (2006). Household technology use: integrating household life and the model of adoption of technology in household. Information Society, 22 (4), 205-218.

Bruer, J. (2010). Can we talk? How the cognitive neuroscience of attention emerged from neurobiology and psychology, 1980-2005. Scientometrics, 83 (3), 751-764.

Bruner, G.; Kumar, A. (2005). Explaining consumer acceptance of handheld Internet device. Journal of Bussiness Reseach, 58 (5), 553-558.

Börner, K.; Chen, C.; Boyack. K. W. (2003). Visualizing knowledge domains. Annual Review of Information Science y Technology. 37 (1), 179-255.

Burton-Jones, A.; Straub, D, W. (2006). Reconceptualizing system usage: An approach and empirical test. Information System Research, 17 (3), 228-246.

Cao, M.; Zhang, Q.; Seydel, J. (2005). B2C e-commerce web site quality: an empirical examination. Industrial Management y Data Systems, 105 (5), 645-661.

Culnan, M. (1986). Management Information System, 1972-1982: A Co-citation Analysis. Management Science, 32 (2), 156-172.

Chen, C. (1998). Generalized similarity analysis and pathfinder network scaling, Interacting with Computers, 10 (2), 107-128.

Chen, C.; Kuljis, J. (2003). The Rising Landscape: A Visual Exploration of Superstring Revolutions in Physics. Journal of the American Society for Information Science and Technology, 54 (5), 435-446.

Chen, C. (2004). Searching for intellectual turning points: Progressive knowledge domain visualization. Proceedings of the National Academy of Sciences of the United States of America, 101 (suppl. 1), 5303-5310.

Chen, T.; Lee, M. (2006). Revealing Themes and Trends in the Knowledge Domain's Intellectual Structure. In A. Hoffman y otros (Eds.), Pacific Rim Knowledge Acquisition Workshop, PKAW 2006 (pp. 99-107). Berlin: Springer.

Cheong, J. H.; Park M. C. (2005). Mobile internet acceptance in Korea. Internet Research, 15 (2), 125-140.

Chuttur, M. Y. (2009). «Overview of the Technology Acceptance Model: Origins, Developments and Future Directions", Indiana University, USA. Sprouts: Working Papers on Information Systems, 9 (37). http://sprouts.aisnet.org/9-37.

Davis, F. (1989). Perceived usefulness, perceived ease of use, and user acceptance of information technology. MIS Quarterly, 13 (3), 319-340. 
Davis, F.; Bagozzi, R. P.; Warshaw, P. R. (1989). User acceptance of computer technology: a comparison of two theoretical models. Management Science, 35 (8), 982-1003.

Edwards, J. R.; Bagozzi, R. P. (2000). On the Nature and Direction of Ralationships between constructs. Psychological Methods, 5 (2), 155-174.

Egghe, L.; Leydesdorff, L. (2009). The Relation between Pearson's correlation Coefficient $\mathrm{r}$ and Salton's Cosine Measure. Journal of the American Society for Information Science and Techology, 60 (5), 1027-1036.

Fishbein, M.; Ajzen, I. (1975). [Online]: Belief, Attitude, Intention and Behavior: An introduction to theory and research. Reading, MA: Addison-Wesley. Available in: <http:// www.people.umass.edu/aizen/f\&a1975.html>.

Freeman, L. (1979). Centrality in networks: Conceptual clarification. Social Networks, 1, 215-239.

Freeman, L.; Borgatti, S.; White, D. (1991). Centrality in valued graphs: A measure of betweenness based on network flow. Social Networks, 13 141-154.

Garfield, E. (1955). Citation indexes for science: A new dimension in documentation through association of ideas. Science, 122 (108-111).

Garfield, E.; Sher, I.; Torpie, R. (1964). The use of citation data in writing the history of science. Philadelphia: Institute for Scientific Information.

Garfield, E. (1994). Scientography: Mapping the tracks of science. Current Contents: Social E Behavioural Sciences, 7 (45), 5-10.

Griffith, B.; Small, H.; Stonehill, J.; Dey, S. (1974). The Structure of Scientific Literatures II: Toward a Macro and Microstructure for Science. Science Studies, 4, 339-364.

Griffith, B. (Ed.) (1980). Key papers in information science (pp. vi-vii). White Plains, NY: Knowledge Industry Publications.

Ha, I.; Yoon, Y.; Choi, M. (2007). Determinants of adoption of mobile games under mobile broadband wireless access environment. Information y Management, 44 (3), 276-286.

Hanneman, R.; Riddle, M. (2005). Introduction to social network methods. Riverside, CA: University of California, Riverside (published in digital form In: <http://faculty.ucr. $\mathrm{edu} /$ hanneman/>.

Henderson, R. M.; Clark, K. B. (1990). Architectural innovation: The reconfiguration of existing product technologies and the failure of estableshed firms. Administrative Science Quarterly, 35, 9-30.

Hong, S. J.; Thong, J. Y. L.; Tam, K. Y. (2006). Understanding continued information technology usage behavior: A comparison of three models in the context of mobile internet. Decision Support Systems, 42, 1819-1834.

Kang, I. S.; Na, S. H.; Lee, S.; Jung, H.; Kim, P.; Sung, W. K.; Lee, J. H. (2009). On coauthorship for author disambiguation. Information Processing and Management, 45, 84-97.

Kim, S.; Malhotra, N. (2005). A longitudinal model of continued IS Use: An integrative view of four mechanisms underlying posadoption phenomena. Management science, 51 (5), 741-755.

King, W. R.; He, J. (2006). A meta-analysis of the technology acceptance model. Information and Management, 43 (6), 740-755. 
Kuhn, T. (1996). The Structure of Scientific Revolutions. (3rd ed.). Chicago: The University of Chicago Press.

Kroenke, D. (2009). Using MIS. $2^{\text {nd }}$ ed. Upper Saddle River, N. J.: Pearson/Prentice Hall. ISBN-10: 0138132488.

Lai, V.; Li, H. (2005). Technology acceptance model for internet banking: an invariance analysis. Information y Management, 42 (2) 373-386.

Lee, M. K. O.; Cheung, C. M. K.; Chen, Z. (2005). Acceptance of Internet-based learning medium: the role of extrinsic and intrinsic motivation. Information y Management, 42 (8), 1095-1104.

Lee, M.; Chen, T. (2009). Trends in Ubiquitous Multimedia Computing. International Journal of Multimedia and Ubiquitous Engineering, 4 (2), 115-124.

Ledezma, R.; Molina, G.; Valero, P. (2002). Análisis de consistencia interna mediante Alfa de Cronbach: un programa basado en gráficos dinámicos. Psico-USF, 7 (2), 143-152.

Legris, P.; Ingham, J.; Collerette, P. (2003). Why do people use information technology? A critical review of the technology acceptance model. Information and Management, 40 (3), 191-204.

Leydesdorff, L.; Wouters, P. (1999). Between texts and contexts: advances in theories of citation. Scientometrics, 44 (2),173-92.

Leydesdorff, L.; Vaughan, L. (2006). Co-occurrence matrices and their applications in information science: Extending ACA to the Web environment. Journal of the American Society for Information Science and Technology, 57, 1616-1628.

Leydesdorff, L. (2008). On the Normalization and Visualization of Author Co-Citation Data: Salton's Cosine versus the Jaccard Index. Journal of the American Society for Information Science and Technology, 59 (1),77-85.

Lin, C, S.; Wu, S.; Tsai, R. J. (2005). Integrating perceived playfulness into expectation-confirmation model for web portal context. Information and Management, 42 (5), 683-693.

Luarn, P.; Lin, H. H. (2005). Toward an understanding of the behavioral intention to use mobile banking. Computers in Human Behavior, 21 (6), 873-891.

Ma, Z.; Yu, K. (2009). Research paradigms of contemporary knowledge management studies: 1998-2007. Journal of Knowledge Management, 14 (2), 175-189.

Malin, B.; Caley, K. (2007). A longitudinal social network analysis of the editorials boards of medical informatics and bioinformatics journals. Journal of the American Medical Informatics Association, 14 (3), 340-348.

Miguel, S.; Moya-Anegón, F.; Solana-Herrero, V. (2007). El análisis de co-citas como método de investigación en Bibliotecología y Ciencia de la Información. Investigación Bibliotecológica, 21 (43), 139-155.

Moya-Anegon, F.; Herrero-Solana, V.; Jimenez-Contreras, E. (2006). A connectionist and multivariate approach to science maps: the SOM, clustering and MDS applied to library science research and information. Journal of Information Science, 32 (1), 63-77.

McCain, K. (1990). Mapping Authors in Intellectual Space: A Technical Overview. Journal of the American Society for Information Science, 41 (6), 433-443.

McCain, K.; Verner, J.; Hislop, G.; Evanco, W.; y Cole, V. (2005). The use of bibliometric and knowledge elicitation techniques to map a knowledge domain: Software Engineering in the 1990s. Scientometrics, 65 (1), 131-144. 
Ngai, E.; Poon, J.; Chan, I. H. (2007). Empirical examination of the adoption of WebCT using TAM. Computers and Education, 48 (2), 250-267.

Nielsen, J. (1993). Usability Enginnering. First Edition. San Francisco, USA: Elsevier. ISBN: 0-12-518406-9.

Nooy, W.; Mrvar, A.; Batagelj, V. (2005). Exploratory social network analysis with Pajek. New York: Cambridge University Press.

Noyons, E.; Van Raan, A. (1998). Monitoring scientific developments from a dynamic perspective: Self-organized structuring to map neural network research. Journal of the American Society for Information Science, 49 (1), 68-81.

Noyons, E.; Moed, H.; Luwel, M. (1999). Combining mapping and citation analysis for evaluative bibliometric purposes: A bibliometric study. Journal of the American Society for Information Science, 50 (2), 115-131.

Nysveen, H.; Pedersen, P.; Thorbjornsen, H. (2005). Intentions to Use Mobile Services: Antecedents and Cross-Service Comparisons. Journal of the Academy of Marketing Science, 33 (3), 330-346.

Peay, E. (1976). A note concerning the connectivity of social networks. Journal of Mathematical Sociology, 4, 319-321.

Petter, S.; Straub, D.; Rai, A. (2007). Specifycing formative constructs in Information System research. MIS Quartery, 31 (4), 623-656.

Pilkington, A.; Meredith, J. (2009). The evolution of the intellectual structure of operations management 1980-2006: A co-citation analysis. Journal of Operations Management, 27(3), 185-202.

Price, D. (1961). Science since Babylon. New Haven: Yale University Press.

Price, D. (1965). Networks of scientific papers. Science, 149, 510-515.

Raghupathi, W.; Nerur, S. (2008). Research and Trends in Health Information Systems. Methods of Information in Medicine, 47 (5), 435-442.

Rosenthal, S.; DiMatteo, M. R. (2001). Meta-analysis: recent developments in quantitative methods form literature reviews, Annual Review of Psychology, 52 (1), 59-82.

Saadé, R.; Bahli, B. (2005). The impact of cognitive absorption on perceived usefulness and perceived ease of use in on-line learning: an extension of the technology acceptance model. Information y Management, 42 (2), 317-327.

Sanchez-Franco, M. J.; Roldan, J. L. (2005). Web acceptance and usage model. A comparison between goal-directed and experiential web users. Internet Research, 15 (1), 21-24.

Sircar, S.; Nerur, P.; Mahapatra, R. (2001). Revolution or Evolution? A Comparison of ObjectOriented and Structured System Development Methods. MIS Quarterly, 25 (4), 457-471.

Shang, R. A.; Chen, Y-C.; Shen, L. (2005). Extrinsic versus intrinsic motivations for consumers to shop on-line. Information y Management, 42 (3), 401-413.

Schepers, J.; Wetzels, M. (2007). A meta-analysis of the technology acceptance model: Investigating subjective norm and moderation effects. Information and Management, 44, 90-103.

Small, H., y Griffith, B. (1974). The Structure of Scientific Literatures I: Identifying and graphing Specialties. Science Studies, 4, 17-40. 
Su, Y. M.; Yang, S. C.; Hsu, P. Y.; Shiau W. L. (2009). Extending co-citation analysis to discover authors with multiple expertise. Expert Systems with Applications, 36, 42874295 .

Sun, H.; Zhang, P. (2006). The role of moderating factors in user technology acceptance. International Journal of Human-Computer Studies, 64 (2), 53-78.

Tang, L.; Walsh, J. (2010). Bibliometric fingerprints: name disambiguation based on approximate structure equivalence of cognitive maps. Scientometrics, 84 (3), 763-784.

Thomson Reuters (2009). Top topics research from map - August 2009. Technology Acceptance Model. Retrieved November, 2010. In: <http://www.vvenkatesh.com/Files/ Sciencewatch.pdf $>$.

Turel, O.; Serenko, A.; Bontis, N. (2007). User acceptance of wireless short messaging services: deconstructing perceived value. Information y Management, 44 (1), 63-73.

Vargas-Quesada, B.; Doménech, I.; García, G., Sanchez, C.; Extremeño, A.; Zulueta, M. (2007). La identificación temática a partir de la visualización de la información: una aproximación mediante el caso de women en Medline. Revista Española de Documentación Científica, 30 (2), 163-177.

Venkatesh, V.; Davis, F. (1996). A model of the antecedents of perceived ease of use: development and test. Decision Sciences, 27 (3), 451-481.

Venkatesh, V.; Morris, M.; Davis, G.; Davis, F. (2003). User acceptance of information technology: towards a unified view. MIS Quarterly, 27 (3), 479-501.

White, H. (1981). Cocited Author Retrieval Online: An Experiment with the Social Indicators Literature. Journal of the American Society for Information Science, 32, 16-22.

White, H.; Griffith, B. (1981). Author Co-citation: A literature Measure of Intellectual Structure. Journal of the American Society for Information Science, 32 (3), 163-172.

White, H.; Griffith, B. (1982). Authors as markers of intellectual space: Co-citation in studies of science, technology and society. Journal of Documentation, 38 (4), 255-272.

White, H.; McCain (1998). Visualizing a Discipline: An Author Co-Citation Analysis of Information Science, 1972-1995. Journal of the American Society for Information Science, 49 (4), 327-355.

White, H. (2003a). Pathfinder Networks and Author Co-citation Analysis: A Remapping of Paradigmatic Information Scientists. Journal of the American Society for Information Science and Technology, 54 (5), 423-434.

White, H. (2003b). Author Co-citation Analysis and Pearson's r, Journal of the American Society for Information Science and Technology, 54 (13), 1250-1259.

Wixon, B. H.; Todd, P. A. (2005). A Theoretical integration of user satisfaction and technology acceptance. Information Systems Research, 16 (1), 85-102.

Wu, J. H.; Wang, S. C. (2005). What drives mobile commerce? An empirical evaluation of the revised technology acceptance model. Information and Management, 42 (5), 719-729.

Yan, X. (1988). On fuzzy cliques in fuzzy networks. Journal of Mathematical Sociology, $13,359-389$. 